\title{
Dynamic modeling and experimental validation for Direct Contact Membrane Distillation (DCMD) process
}

\author{
Fadi Eleiwi ${ }^{1}$, Noreddine Ghaffour ${ }^{2}$, Ahmad S. Alsaadi ${ }^{2}$, Lijo Francis ${ }^{2}$, \\ Taous Meriem Laleg-Kirati ${ }^{1 \dagger}$ \\ ${ }^{1}$ Computer, Electrical and Mathematical Science and Technology Department (CEMSE), \\ King Abdullah University of Science and Technology (KAUST), Thuwal, Saudi Arabia \\ ${ }^{2}$ Water Desalination and Reuse Center (WDRC), Biological and Environmental Science \\ and Engineering Department (BESE), King Abdullah University of Science and \\ Technology (KAUST), Thuwal, Saudi Arabia
}

\begin{abstract}
This work proposes a mathematical dynamic model for the direct contact membrane distillation (DCMD) process. The model is based on a 2D Advection-Diffusion Equation (ADE), which describes the heat and mass transfer mechanisms that take place inside the DCMD module. The model studies the behavior of the process in the time varying and the steady state phases, contributing to understanding the process performance, especially when it is driven by intermittent energy supply, such as the solar energy. The model is experimentally validated in the steady state phase, where the permeate flux is measured for different feed inlet temperatures and the maximum absolute error recorded is $2.78^{\circ} \mathrm{C}$. Moreover, experimental validation includes the time variation phase, where the feed inlet temperature ranges from $30^{\circ} \mathrm{C}$ to $75^{\circ} \mathrm{C}$ with $0.1^{\circ} \mathrm{C}$ increment every 2 minutes. The validation marks relative error to be less than $5 \%$, which leads to a strong correlation between the model predictions and the experiments.
\end{abstract}

Keywords: Direct contact Membrane Distillation (DCMD); Dynamic modeling; 2D Advection-Diffusion Equation (ADE) model; Discretization; Intermittent energy supply.

\footnotetext{
${ }^{1 \dagger}$ Corresponding author Tel.: +966-12-808 3428.

Email:taousmeriem.laleg@kaust.edu.sa (T.M. Laleg-Kirati).
} 


\section{Introduction}

Membrane distillation (MD) is a thermal separation process that utilizes a specific type of membranes for different applications, such as water desalination. Membranes are selected to be micro porous, non-wetted, and highly hydrophobic [1-4]. Such properties allow the transfer of only water vapor or other volatile molecules through the membrane dry pores, and produce clean water. The separation is achieved under the validation of vapor-liquid equilibrium principle for molecules separation [3]. MD is an attractive technology, with promising potentials to take the lead over a wide range of other existing water desalination methods, such as multi-stage flash (MSF) and reverse osmosis ( $\mathrm{RO})[4-7]$. These potentials contribute toward promoting the MD process to be a cost-effective desalination process through the capabilities to be powered with renewable energy sources, such as geothermal, solar energy or low grade waste heat [8-10].

The process starts when the solution in the feed side, after being heated to a desired temperature, evaporates along the membrane boundary layer. The vapor passes through the membrane dry pores, driven by the difference of pressure between the two sides of the membrane maintained by the difference of the temperature, and gets condensed in the permeate side providing clean water $[6,11,12]$.

Permeate flux in the MD process depends on the vapor gradient pressure between the two sides of the membrane, in addition to the characteristics of the module and the membrane. Four common configurations, mainly airgap membrane distillation (AGMD), vacuum membrane distillation (VMD), sweeping-gas membrane distillation (SGMD), and direct contact membrane distillation (DCMD), are proposed and widely studied [3]. Though other configurations are also developed such as water gap MD [13]. Vapor condensation which occurs in the permeate side is the main difference among these configurations [14].

Many theoretical studies have been carried out to model and predict the performance of the MD process. Such studies contribute further towards deep understanding of the involved parameters and enhancing the performance of the process. The proposed models are mainly empirical, and hold the advantages of relating the involved process parameters with each other to show the effect of the overall performance. The majority of the proposed models agreed on considering the steady state performance to be more important than the time varying one $[3,15,16]$. These studies adopted the kinetic the- 
ory of gases through porous media in their models $[4,6,7]$, and studied the performance of the MD processes based on the selected MD configuration. Of interest, the proposed models lacked the compatibility to be powered with intermittent energy supply, such as the solar energy source. This is because they could not accommodate the unsteady nature of the intermittent energy sources $[17,18]$. Notwithstanding, some studies proposed the use of heating storage tanks as a remedy to the intermittent unsteadiness, however they introduced additional problems related to the incompatibility of the space requirement for some applications [19], and the extra expenses they require for thermal insulation and maintenance [20], beside the relatively low storage capacity they provide [21], to list few.

Enhancing the productivity of the MD process accompanied with deep understanding of its behavior is the motivation of this paper, so we propose a mathematical dynamic model for the DCMD process with experimental validation in both time varying and steady state phases. The model is based on a 2D Advection-Diffusion Equation (ADE), and it is considered to be in two dimensions to include the dimensions of the membrane as well as the feed and the permeate solutions. The significance of the model appears when it contributes toward improving the performance of the process and saves energy consumption. The model predicts the process to be powered with intermittent energy supply; considering its unsteady nature. Moreover, it contributes for an accurate description of the time evolution for the different heat transfer mechanisms inside the MD module, and accounts for sudden changes which might happen to the process. All of these features lead eventually to make the process more suitable for automation and control systems $[22,23]$, and therefore, better performance and efficiency.

The model is set for the DCMD configuration due to its convenience for laboratory tests and simplicity [11], but it can be extended to include other MD configurations.

\section{Theory}

\subsection{Heat and mass transfer mechanisms in MD process}

Improving the performance of the MD process needs a comprehensive knowledge of the different heat and mass transfer mechanisms which drive it. 


\subsubsection{Mass transfer}

Maintaining the permeate solution to be cooler than the feed solution forces vapor molecules to transfer through the membrane dry pores, and this happens from the feed side to the permeate side where it condenses.

Mass transfer starts when the feed solution reaches the evaporation limit, then vapor is formed in a small region of the membrane boundary layer. The vapor then is ready to pass the dry pores of the membrane toward the permeate side. The transfer is driven by the vapor pressure gradient between the feed solution and the permeate solution.

Mass transfer is directly proportional to the permeability of the membrane as well as the vapor pressure gradient through the permeate mass flux as expressed in Eq.(1) [24].

$$
\mathcal{J}(T)=B(T)\left(P_{m f}(T)-P_{m p}(T)\right),
$$

where $\mathcal{J}(T)$ is the permeate mass flux, $P_{m f}(T), P_{m p}(T)$ are the interfacial pressure along the membrane boundaries with the feed solution and the permeate solution, respectively. $B(T)$ is the membrane mass transfer coefficient of the process, which is usually modeled with either Knudsen model, Poiseuille model or a combination of them in the so-called a molecular model [25]. Knudsen model is most suitable to describe the mass transfer mechanism in the DCMD process, since its criteria of having a mean free path of the transferred molecules to be larger than the membrane pore size is achieved [3], and the effect of the molecular diffusion for the air trapped within each membrane pore is extremely small comparing to the water flux [3, 25-27], therefore it is neglected. The formula of the mass transfer coefficient is expressed as follows:

$$
B(T)=1.064 \frac{r \epsilon}{\chi \delta_{m}}\left(\frac{M}{R T_{\text {mean }}}\right)^{0.5} .
$$

where $r$ is the pore size, $\epsilon$ is the porosity, $\chi$ is the tortuosity, $\delta_{m}$ is the membrane thickness, $R$ is the gas universal constant, $M$ is the water molecule weight, $T_{\text {mean }}$ is the mean temperature along the membrane interfaces.

It is worth mentioning that Knudsen model requires full knowledge of some membrane parameters, such as pore size, porosity and thickness [26].

\subsubsection{Heat transfer}

Heat transfers with the vapor and volatile molecules from the feed solution to the permeate solution. This transfer is following the second law of 
thermodynamics, in which the transferred heat takes a form of sensible and latent heat inside the membrane, and conduction as well as convection heat transfer throughout the bulk flow in the feed and the permeate solutions $[17,18]$.

The majority of the heat transfer models are set according to some factors, such as heat balance equations, and conservation of energy and mass laws in thermodynamics. As a result, the involved amount of transferred heat should be equal in both process solutions as well as through the membrane dry pores. Fig.1 shows a schematic diagram for the heat transfer mechanisms that take place during a standard operation of an MD process.

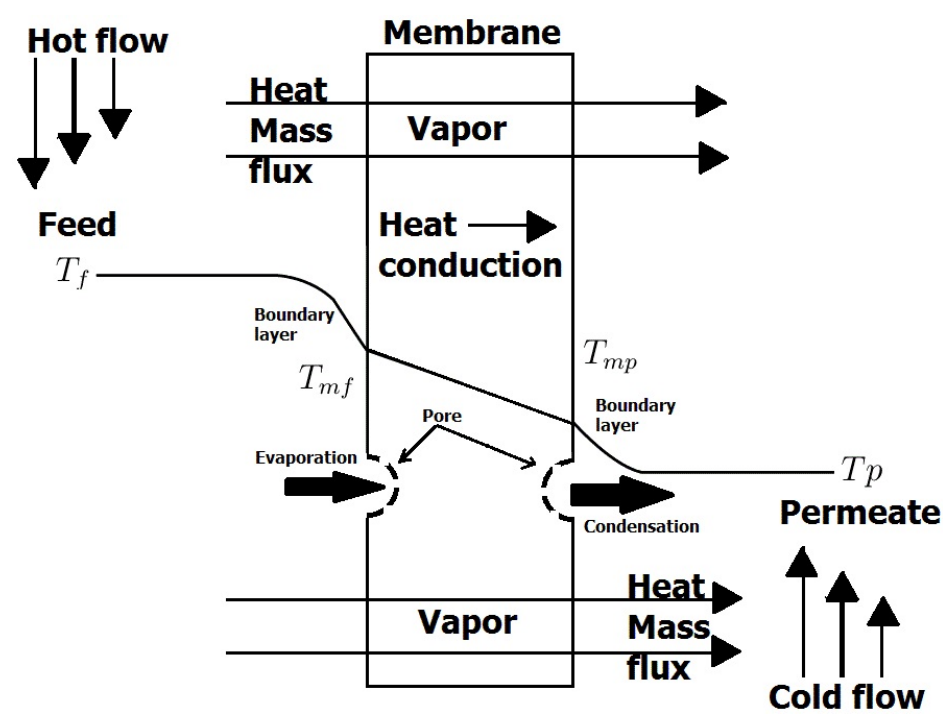

Figure 1: Different heat transfer mechanisms in MD process.

Following Eqs (3),(4), and (5) express the involved amount of generated and transferred heat in the process [28].

$$
\begin{aligned}
Q_{f} & =A \nu_{f}\left(T_{f}-T_{m f}\right), \\
Q_{p} & =A \nu_{p}\left(T_{m p}-T_{p}\right), \\
Q_{m} & =\frac{A k_{m}}{\delta_{m}}\left(T_{m f}-T_{m p}\right)+A \mathcal{J}(T) H(T) .
\end{aligned}
$$


and the energy balance equations are:

$$
A \nu_{f}\left(T_{f}-T_{m f}\right)=\frac{A k_{m}}{\delta_{m}}\left(T_{m f}-T_{m p}\right)+A \mathcal{J}(T) H(T)=A \nu_{p}\left(T_{m p}-T_{p}\right) .
$$

where $Q_{f}, Q_{p}, Q_{m}$ are the total heat transferred from the feed through the membrane to the permeate, $k_{m}$ is the average thermal conductivity of the membrane and vapor, $A$ is the membrane area. $\nu_{f}, \nu_{p}$ are the convective heat transfer coefficients on the feed and the permeate solutions, respectively. $H(T)$ is the latent heat of evaporation which is responsible of the admissible sensible heat.

$$
H(T)=2500.8-2.36 T+0.0016 T^{2}-0.00006 T^{3} .
$$

\subsubsection{Existing models for DCMD}

MD modeling literature is rich with many models which have been developed to describe different phenomena at the membrane boundary layers level. However, the majority of these models were built over empirical relations and laboratory experiments. These models are either valid for steady state phase, or considers only zero-order performance with no dynamic behavior. This means that their validity is only after sufficient period of time, where the process reaches the steady state phase and the performance becomes stationary. In $[3,29]$ a group of mathematical relations for the temperature of the membrane boundary layers region are proposed. These relations are derived from semi-empirical relations and laboratory experiments. Moreover, these relations are based on the fact that it is difficult to measure interfacial temperatures and concentrations directly. In addition, another model is proposed in [3] to estimate those parameters considering a full knowledge of the heat and mass transfer coefficients throughout the MD module and bulk flow temperatures and concentrations. In [15] a group of differential equations with respect to space is assumed. The model gives a formula for the membrane interfaces temperature by relating some parameters of the process. However it doesn't count for the time evolution of the process. This model is similar to the model in [3] with additional complexity. In [16] more elaboration is spent on the same empirical relations that $[3,15]$ used. The contribution is through a development of an optimized iterative method that reduces the error between initial guess for the membrane interfaces temperature with the assumed values, so they succeeded to know the temperature in each experiment. In [7] an intuition to consider a dynamic model for time evo-

lution of the heat in the MD process was addressed, where the interest was 
focused on introducing numerical simulations for Navier-Stokes equations. Later on, the interest in dynamic modeling for the MD process continued, and followed with many studies that tackled the dynamic analysis of heat and mass transfer using Computational Fluid Dynamics (CFD) techniques [30-34]. For more numerical models for the MD process see [35-40]

Literature models offer a predictive estimation for the temperature of the boundary layer region of the membrane, however they are unable to provide information about the temperature of the bulk solutions in the feed and the permeate sides. Additionally, the steady state models do not count for the time evolution of the process, and the needs of the intermittent sources, therefore they can not capture any sudden changes which might happen to the process. For example, if we consider the use of solar energy source to drive the MD process, we need the model to count for the variable output intensity of the source throughout the day. Moreover, CFD modeling requires intensive computational effort and cost.

\subsection{Dynamic modeling of DCMD}

In this section, we propose a mathematical dynamic model for the DCMD process, which considers the time evolution of the heat and mass transfer mechanisms throughout the feed and the permeate solutions. In addition, the model offers a complete scene to the process performance starting with the transient behavior of the process until reaching the steady state phase.

The significance of the model appears when it allows the process to be powered with renewable energy sources in an efficient manner (save energy). Moreover, it contributes toward improving the performance of the process through an accurate description of the time evolution of the different heat and mass transfer mechanisms inside the MD module. Additionally, the dynamic model is suitable for supervision and detection of any fault which might happen in the process. These features of the model help the process for automation and control systems in later stages.

Heat inside feed and permeate solutions transfers in a form of a sensible and latent heat, and convective heat. Therefore, mechanisms of heat conduction and heat convection are essential to be considered within the study of heat evolution inside a DCMD module. Fig.2 shows a schematic diagram

of a counter current DCMD module with the coordinates arrangement for each process component. 


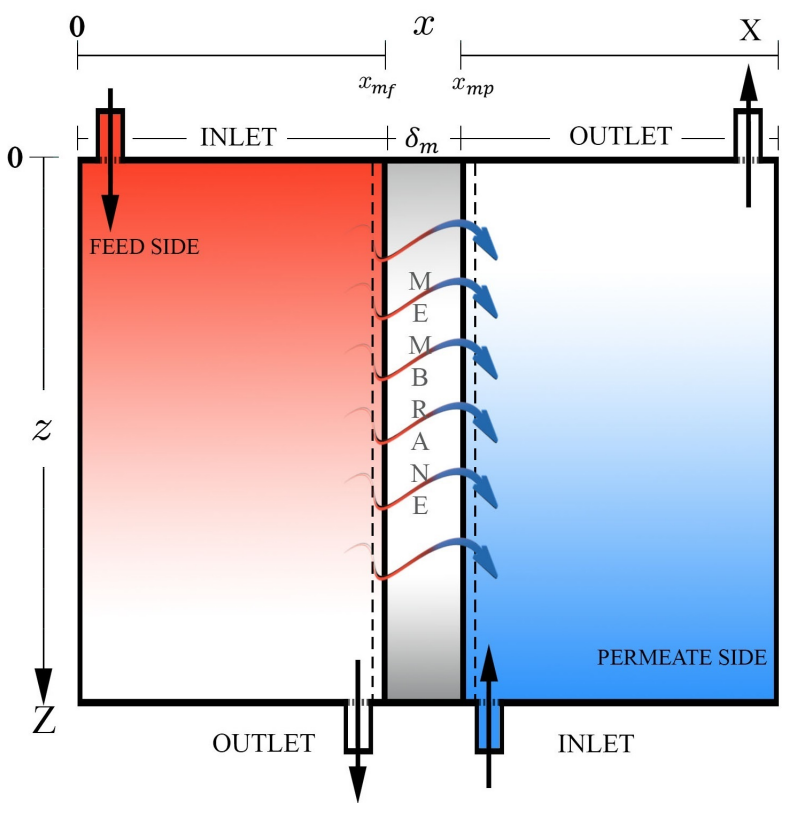

Figure 2: DCMD counter current schematic diagram with the inlets and outlets of hot/cold water.

Due to the very small thickness of the used membranes, heat transfer mechanisms inside the feed and the permeate solutions are affected by each other, and coupled together. This attachment is clear via the heat flux that flows from the feed to the permeate through the dry pores of the membrane. Therefore, we propose the use of a 2D ADE to model the different heat and mass transfer mechanisms inside the MD process $[17,18]$. The interesting properties of the ADE model make it possible to describe the convection and conduction mechanisms of heat transfer inside feed and permeate solutions. Moreover, the model counts for the time evolution of MD process behavior.

In the proposed model, we assume that the convective heat mechanism is dominant along the length of the membrane (namely the $z$-axis), and the conduction heat mechanism which is responsible of transferring the heat through the membrane dry pores (heat diffusion) is dominant along the direction toward the membrane (namely the $x$-axis). Through these assumptions, we consider the flow rate in the $z$-axis to be constant during the operation of the process, while neglecting the flow rate in the $x$-axis. 


\subsubsection{DCMD Mathematical model}

The mathematical model of the DCMD module is presented in Eq.(8), where the domain of interest is rectangular with finite values for the time, and the space coordinates for each subsystem container as shown in Fig.2.

$\frac{\partial T(x, z, t)}{\partial t}+v_{z}^{k} \frac{\partial T(x, z, t)}{\partial z}+v_{x}^{k} \frac{\partial T(x, z, t)}{\partial x}=\alpha^{k}\left(\frac{\partial^{2} T(x, z, t)}{\partial x^{2}}+\frac{\partial^{2} T(x, z, t)}{\partial z^{2}}\right)$

with

$$
k=\{f, p\}, \quad 0<t<\tau_{\text {final }}, \quad x \in\left[0, x_{f m}\right] \cup\left[x_{p m}, X\right], \quad 0<z<Z,
$$

where $k=\{f, p\}$ refers to the feed and the permeate, respectively. $v_{x}^{k}$ and $v_{z}^{k}, k=\{f, p\}$ are the feed and permeate pumps flow rates along the $x$ and $z$ coordinates, respectively. According to our assumption, the fluid flows only along the vertical coordinate such as $v_{z}^{k}$, and therefore the horizontal flow is neglected such as $v_{x}^{k}=0$. Additionally, $\alpha^{k}, k=\{f, p\}$ is the feed and the permeate thermal diffusivity constant. It is assumed that the horizontal diffusivity is more affecting and has more significance than the vertical one, therefore the vertical thermal diffusivity is assumed to be negligible. The thermal conductivity constant of the used water, $k$, water specific heat, $c_{p}$, and the density, $\rho$ of the water used, as follows $\frac{k}{\rho c_{p}}[41,42]$.

The 2D ADE model in Eq.(8) is rewritten in Eq.(9) after considering the above assumption.

$$
\frac{\partial T(x, z, t)}{\partial t}+v_{z}^{k} \frac{\partial T(x, z, t)}{\partial z}=\alpha_{x}^{k} \frac{\partial^{2} T(x, z, t)}{\partial x^{2}},
$$

with

$$
k=\{f, p\}, \quad 0<t<\tau_{\text {final }}, \quad x \in\left[0, x_{f m}\right] \cup\left[x_{p m}, X\right], \quad 0<z<Z,
$$

The initial conditions of the model are

$$
T_{f}(x, z, 0)=T_{f_{\text {initial }}}, T_{p}(x, z, 0)=T_{p_{\text {initial }}}
$$

where the associated boundary conditions with the dynamic model (e.g. feed solution) are set to be a Dirichlet condition for the inlet temperature of 
each subsystem, and Neumann conditions for the rest of the boundaries like Eq.(11).

$$
\begin{cases}T(x, 0, t) & =T_{0_{f}} \text { for } x \in\left[0, x_{m f}\right], \\ T(x, Z, t) & =T_{0_{p}} \text { for } x \in\left[x_{m p}, X\right] \\ \left.\frac{\partial T(x, z, t)}{\partial x}\right|_{x=0} & =0,\left.\quad \frac{\partial T(x, z, t)}{\partial x}\right|_{x=X}=0 \\ \left.\frac{\partial T(x, z, t)}{\partial x}\right|_{x=x_{m f}} & =\left[\mathcal{J}(T) H(T)+\frac{k_{m}}{\delta_{m}} T\left(x_{m p}, z, t\right)\right. \\ & \left.-\frac{k_{m}}{\delta_{m}} T\left(x_{m p}, z, t\right)\right] / k_{f}, \\ \left.\frac{\partial T(x, z, t)}{\partial x}\right|_{x=x_{m p}} & =\left[\mathcal{J}(T) H(T)+\frac{k_{m}}{\delta_{m}} T\left(x_{m f}, z, t\right)\right. \\ & \left.-\frac{k_{m}}{\delta_{m}} T\left(x_{m p}, z, t\right)\right] / k_{p}, \\ \left.\frac{\partial T(x, z, t)}{\partial z}\right|_{z=Z} & =\phi,\left.\quad \frac{\partial T(x, z, t)}{\partial z}\right|_{z=0}=\phi\end{cases}
$$

where $k_{f}, k_{p}$ are the thermal conductivity coefficient of the feed and the permeate solutions, respectively. $\phi \in \mathbb{R}^{+}$is a positive constant to represent the amount of flux that is released outside the process containers. In this paper, we consider the amount of released flux outside the process containers to be zero, such as $\phi=0$.

\subsubsection{Semi-discretized model}

It is usually difficult to obtain analytic solutions for Partial Differential Equations (PDEs) models, hence numerical methods are the appropriate alternative to get an acceptable approximation for these solutions. In the numerical implementation, the 2D ADE model is semi-discretized, where convection and conduction are considered to be effective along the $x, z$-axes. The convective and diffusive terms are solved with first-order upwind scheme, and central differencing scheme, respectively. The upwind scheme warrants the stability, and ensures the solution to satisfy the entropy condition [43], as in Eq.(12).

$$
\frac{\partial T(x, z, t)}{\partial z}=\frac{T_{i, j+1}-T_{i, j}}{\Delta z}
$$

where where the variable $T_{i, j}$ represents the temperature distribution at the grid point $(i, j)$ inside the feed and the permeate domain, and $\Delta z$ is the spatial step size along the $z$-axis. On the same context, the central difference 
scheme is suitable for a second derivative diffusive terms [43] like in Eq.(13).

$$
\frac{\partial^{2} T(x, z, t)}{\partial x^{2}}=\frac{T_{i+1, j}-2 T_{i, j}+T_{i-1, j}}{\Delta x^{2}} .
$$

where $\Delta x$ is the spatial step size along the $x$-axis.

The relations of the grid points in the domain are significant to solve the model. Fig. 3 shows the grid of discretization, where the points $T_{i, j} \in$ $\left[0, x_{m f}\right]$ represent the temperature distribution of the feed solution and the points $T_{i, j} \in\left[x_{m p}, X\right]$ represent the temperature distribution of the permeate solution. It is worth mentioning that in DCMD the permeate solution starts right after the thickness of the membrane and the width of the feed solutions as in Eq.(14).

$$
x_{m p}=x_{m f}+\delta_{m} .
$$

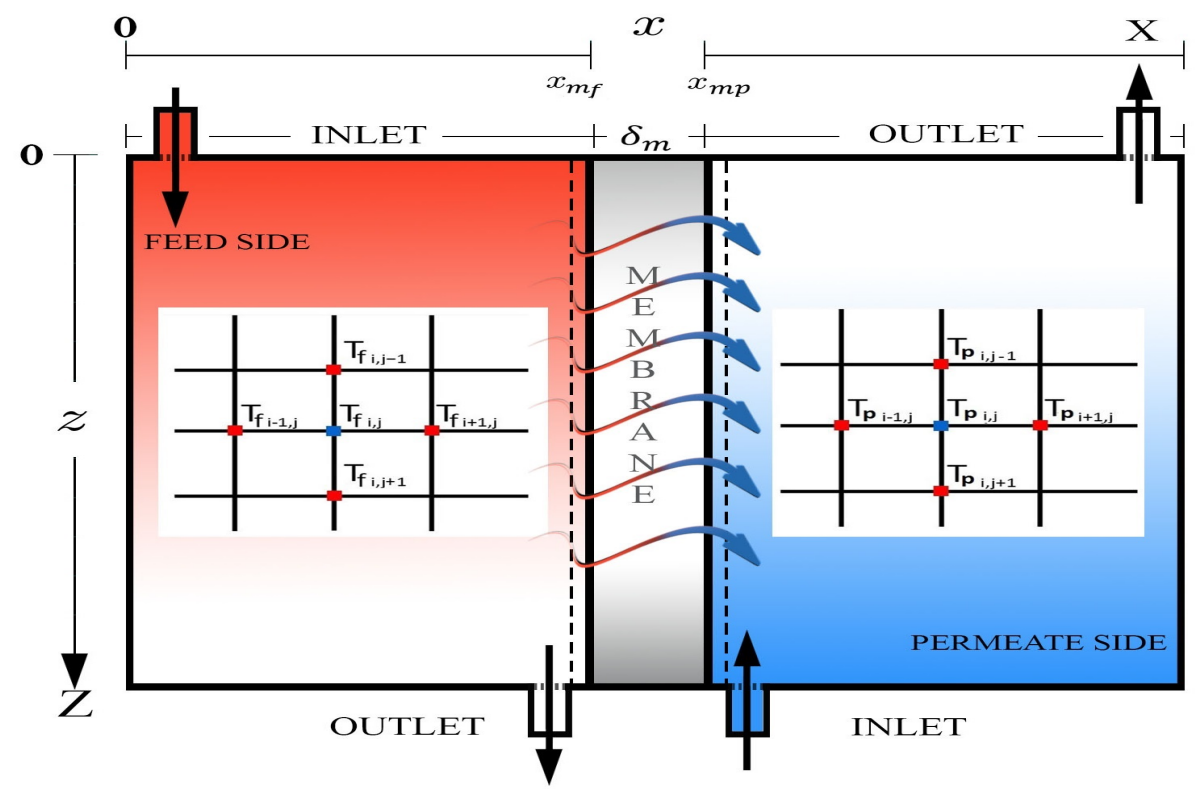

Figure 3: Discretization grid for the feed and the permeate.

The semi-discrete version of the continuous 2D ADE model is a nonlinear high dimension system of ODEs because of the nonlinearity of the boundary 
conditions, following is the model.

$$
\left\{\begin{aligned}
\dot{\mathbf{T}}(t) & =\mathbf{A} \mathbf{T}(t)+\mathbf{B u}(t)+f(\mathbf{T}(t)) \\
\mathbf{y} & =\mathbf{C} \mathbf{T} \\
\mathbf{T}(\mathbf{0}) & =\mathbf{T}_{0}
\end{aligned}\right.
$$

where $\mathbf{T} \in \mathbb{R}^{2 n^{2} \times 1}$ is the state vector, which represents the time evolution of the temperature distribution at each grid point inside the feed and the permeate designated domain $\mathbf{T}_{f}, \mathbf{T}_{p}$, respectively.

$$
\mathbf{T}=\left[\begin{array}{c}
\mathbf{T}_{f} \\
\mathbf{T}_{p}
\end{array}\right]
$$

where $\mathbf{T}_{f}, \mathbf{T}_{p} \in \mathbb{R}^{n^{2} \times 1}$ and $n$ is the number of discretization points in $x$ and $z$ axes, and $\mathbf{T}_{0}$ is the initial condition of the state vector. Matrix $\mathbf{A} \in \mathbb{R}^{2 n^{2} \times 2 n^{2}}$ represents the dynamics matrix which includes all dynamics of the feed and the permeate with their correspondent couplings on the boundaries, as in Eq.(17).

$$
\mathbf{A}=\left[\begin{array}{cc}
\mathbf{A}_{f_{i, j}} & C_{f p} \\
C_{p f} & \mathbf{A}_{p_{i, j}}
\end{array}\right],
$$

where $i, j$ are the discretization grid indices.

$$
\left\{\begin{array}{l}
A_{f_{i, j}\left(v_{f}\right)}=\left(-\frac{v_{z}^{f}}{\Delta z}-\frac{2 \alpha_{x}^{f}}{\Delta x^{2}}\right) T_{i, j}+\left(\frac{v_{z}^{f}}{\Delta z}\right) T_{i, j-1}+\left(\frac{\alpha_{x}^{f}}{\Delta x^{2}}\right) T_{i-1, j}+\left(\frac{\alpha_{x}^{f}}{\Delta x^{2}}\right) T_{i+1, j} \\
A_{p_{i, j}\left(v_{p}\right)}=\left(\frac{v_{z}^{p}}{\Delta z}-\frac{2 \alpha_{x}^{p}}{\Delta x^{2}}\right) T_{i, j}-\left(\frac{v_{z}^{p}}{\Delta z}\right) T_{i, j+1}+\left(\frac{\alpha_{x}^{p}}{\Delta x^{2}}\right) T_{i-1, j}+\left(\frac{\alpha_{x}^{p}}{\Delta x^{2}}\right) T_{i+1, j} \\
C_{f p}=\frac{\alpha_{x}^{f}}{\Delta x^{2}} \frac{\Delta x k_{m}}{\delta_{m} k_{f}} \\
C_{p f}=\frac{\alpha_{x}^{p}}{\Delta x^{2}} \frac{\Delta x k_{m}}{\delta_{m} k_{p}}
\end{array}\right.
$$

$\mathbf{B}$ is the input matrix in Eq.(19), and $\mathbf{u}(t)$ includes the inputs of the DCMD process such as in Eq.(20). 


$$
\mathbf{B}=\left[\begin{array}{llc}
\left(\frac{v_{z}^{f}}{\Delta z}\right)_{1} & 0 & \\
\vdots & & \vdots \\
\left(\frac{v_{z}^{f}}{\Delta z}\right)_{n} & 0 & \\
0 & & 0 \\
\vdots & & \vdots \\
0 & & 0 \\
0 & \left(-\frac{v_{z}^{p}}{\Delta z}\right)_{1} & \\
0 & & \vdots \\
0 & \left(-\frac{v_{z}^{p}}{\Delta z}\right)_{n}
\end{array}\right], \quad(19) \quad \mathbf{u}(t)=\left[\begin{array}{l}
T_{0_{f}} \\
T_{0_{p}}
\end{array}\right]
$$

$f(\mathbf{T}) \in \mathbb{R}^{2 n^{2} \times 1}$ contains the different boundary conditions of the feed and the permeate, especially the ones associated with the membrane boundaries. Some of the boundary conditions are nonlinear and functions of the state vector $\mathbf{T}$ as shown in Eq.(11). Vectors $\zeta$ and $\eta$ in Eqs.(22) and (23) represent the polynomial nonlinearity structure in $f(\mathbf{T})$.

$$
\begin{gathered}
f(\mathbf{T})=\left[\zeta_{1} \cdots \zeta_{n} \eta_{1} \cdots \eta_{n}\right]^{T}, \\
\zeta=\left[0 \cdots 0 \frac{\alpha_{x}^{f}}{\Delta x} \frac{\mathcal{J}(T) H(T)}{k_{f}}\right]^{T}, \\
\eta=\left[-\frac{\alpha_{x}^{p}}{\Delta x} \frac{\mathcal{J}(T) H(T)}{k_{p}} 0 \cdots 0\right]^{T} .
\end{gathered}
$$

Finally, we are measuring only the two outlet temperatures in the output vector $\mathbf{y} \in \mathbb{R}^{2 \times 1}$ in Eq. $(24)$

$$
\mathbf{y}=\left[\begin{array}{c}
T_{\text {fout }} \\
T_{\text {pout }}
\end{array}\right]
$$

with the observation matrix $\mathbf{C} \in \mathbb{R}^{2 \times 2 n^{2}}$ in Eq.(25).

$$
\mathbf{C}=\left[\begin{array}{ccc}
\overbrace{0 \cdots 0}^{n^{2}} & \overbrace{10 \cdots 0}^{n-1} & \overbrace{00 \cdots 0}^{n(n-1)+1} \\
0 \cdots \cdots & 0 \cdots \cdots 0 & 10 \cdots 0
\end{array}\right] .
$$




\section{Experiments}

A fully automated DCMD experimental lab scale set-up was locally designed and fabricated at King Abdullah University of Science and Technology (KAUST) workshop [10]. A schematic diagram of the experimental set-up is presented in Fig.4. A flat sheet membrane module with an active surface area of $0.005 \mathrm{~m}^{2}$, with flow channel dimensions of $0.1 \mathrm{~m} \times 0.05 \mathrm{~m} \times 0.002 \mathrm{~m}$, was designed and fabricated using poly methyl methacrylate (PMMA) material. A composite membrane with a poly tetra fluoroethylene (PTFE) active layer and a nonwoven poly propylene support layer was used for MD testing. Details of the membrane characteristics and its performance for seawater desalination were widely reported in previous works, e.g. $[5,10,13]$. Some of these parameters are presented in Table 1, while Table 2 presents the feed water characteristics.

Table 1: Characteristics of the membrane.

\begin{tabular}{l|c}
\hline \hline Parameter & Value \\
\hline \hline Membrane type & PTFE \\
\hline Liquid Entry Pressure $(P S I)$ & 15 \\
\hline Thickness $(\mu \mathrm{m})$ & $170 \pm 3$ \\
\hline Tortuosity & 1.35 \\
\hline Mean flow pore size $(\mu \mathrm{m})$ & 0.26 \\
\hline First bubble point $(\mu \mathrm{m})$ & 0.42 \\
\hline Porosity $(\%)$ & $73 \pm 4$ \\
\hline Contact angle & $140{ }^{\circ} \mathrm{C} \pm 3^{\circ} \mathrm{C}$ \\
\hline $\begin{array}{c}\text { Mass Transfer Coefficient } \\
\left(\mathrm{kg} / \mathrm{h} . \text { Pa. } \mathrm{m}^{2}\right)\end{array}$ & 0.01 \\
\hline \hline
\end{tabular}


Table 2: Characteristics of the feed water (Redseawater).

\begin{tabular}{l|c}
\hline \hline Parameter & Feed water(Redseawater) \\
\hline \hline $\mathrm{pH}$ & 8.03 \\
\hline Conductivity $(\mu \mathrm{S} / \mathrm{cm})$ & 61,400 \\
\hline Turbidity $(N T U)$ & 0.96 \\
\hline TOC $(\mathrm{mg} / \mathrm{L})$ & 1.94 \\
\hline Calcuim $(\mathrm{mg} / \mathrm{L})$ & 480.5 \\
\hline Magnesium $(\mathrm{mg} / \mathrm{L})$ & 1530 \\
\hline Sodium $(\mathrm{mg} / \mathrm{L})$ & 12,628 \\
\hline Boron $(\mathrm{mg} / \mathrm{L})$ & 5.1 \\
\hline Potassium $(\mathrm{mg} / \mathrm{L})$ & 425 \\
\hline Chloride $(\mathrm{mg} / \mathrm{L})$ & 23,128 \\
\hline Sulfate $(\mathrm{mg} / \mathrm{L})$ & 2650 \\
\hline \hline
\end{tabular}

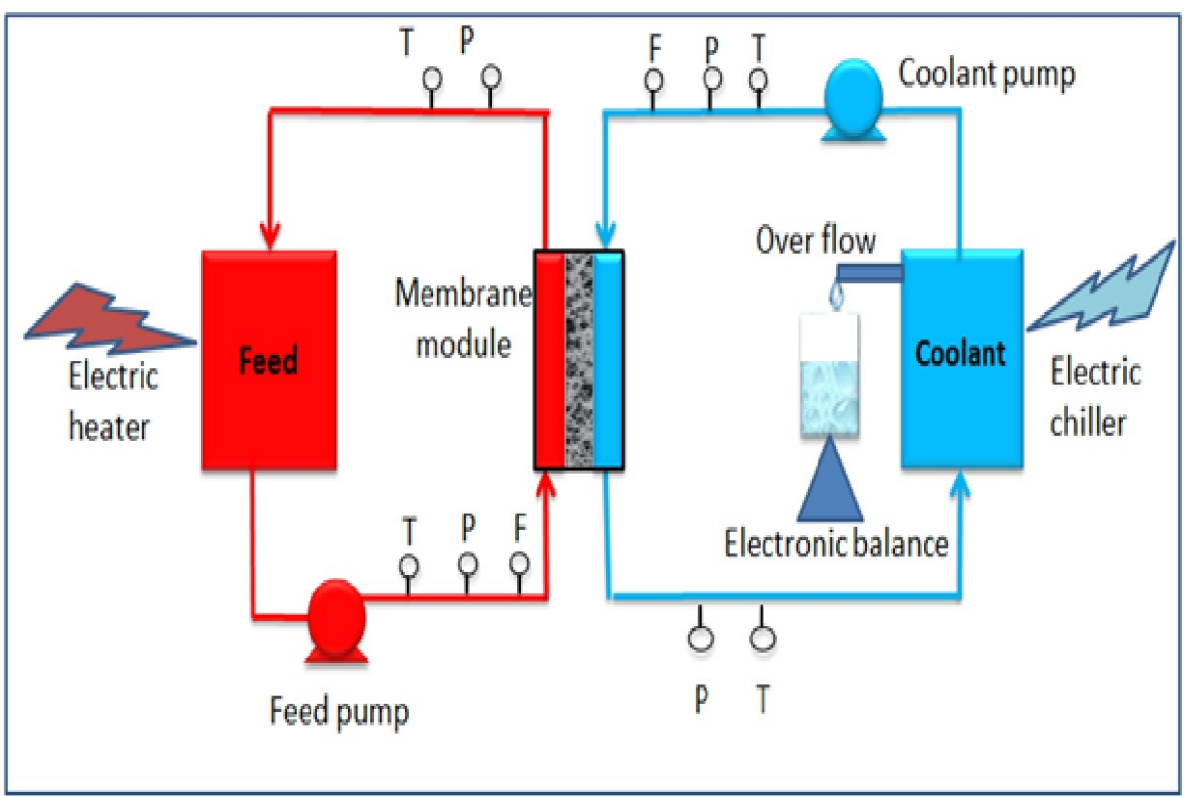

Figure 4: A schematic diagram of the experimental lab scale set up.

Red Sea water was preheated to desired temperatures and circulated through the feed side of the membrane, while de-ionized (DI) water was circulated through the other side of the membrane simultaneously in a counter- 
current mode. Feed and permeate temperatures were controlled using thermoregulators. Fresh seawater was used for each experiment. The permeate generated during the MD process results in an increase in the volume of coolant and overflow can happen through the outlet of the permeate/coolant tank. The overflown permeate was collected in a separate container placed on a weighting balance. The increase in weight of the container was continuously monitored and recorded. Pressure, temperature and flow rates were continuously monitored at the inlet and outlet of the module of both feed and permeate sides using respective sensors connected to the data acquisition unit equipped with a Lab View Software. Conductivities of both permeate and feed solutions were continuously monitored and measured using conductivity meters (Oakton Eutech Instruments, Malaysia).

The influence of feed water temperature ranging from $40^{\circ} \mathrm{C}$ to $70^{\circ} \mathrm{C}$ on water vapor flux was studied by maintaining a constant temperature on the permeate side at $20^{\circ} \mathrm{C}$. All the data were recorded after reaching the steady state temperature. Similarly, the influence of feed water temperature ranging from $30^{\circ} \mathrm{C}$ to $75^{\circ} \mathrm{C}$ on trans- membrane flux was also studied by ramping the feed solution temperature at a rate of $0.1^{\circ} \mathrm{C}$ per 2 minutes. During ramping experiments, the coolant temperature was also maintained at $20^{\circ} \mathrm{C}$. Feed and coolant flow rates were kept constant at $90 \mathrm{~L} / \mathrm{h}$ and $60 \mathrm{~L} / \mathrm{h}$, respectively, for all the experiments.

The salt rejection was calculated by: $S R=\left(1-C o_{p} / C o_{f}\right) * 100$, where $C o_{p}$ and $\mathrm{Co}_{f}$ are the salt concentrations of permeate and feed solutions, respectively. A salt rejection of $99.99 \%$ has been observed in all experiments. The water vapor flux was measured by: $\mathcal{J}=m w / A t$, where $m w$ is the weight of collected permeate at a particular time interval $t$, and $A$ is the effective membrane area.

\section{Results and discussion}

\subsection{Model validation}

Simulations of the ADE model have been conducted with a developed MATLAB ${ }^{\circledR}$ software, where it is a very useful tool which has proven success in simulating mathematical models for different physical phenomena, and performs comprehensive analysis studies for a wide range of applications (see $[44,45]$ ). The ADE model, then, has been validated with experimental data through two sets of experiments. Of great interest and for validation purposes, the same DCMD parameter values, membrane specifications, and 
environment conditions have been applied for the experiments and the numerical simulations.

\subsubsection{Steady state validation}

Fig.5 shows the validation at the steady state phase between the experimental data and the numerical simulations for different feed inlet temperatures. The absolute error is also presented in the figure.

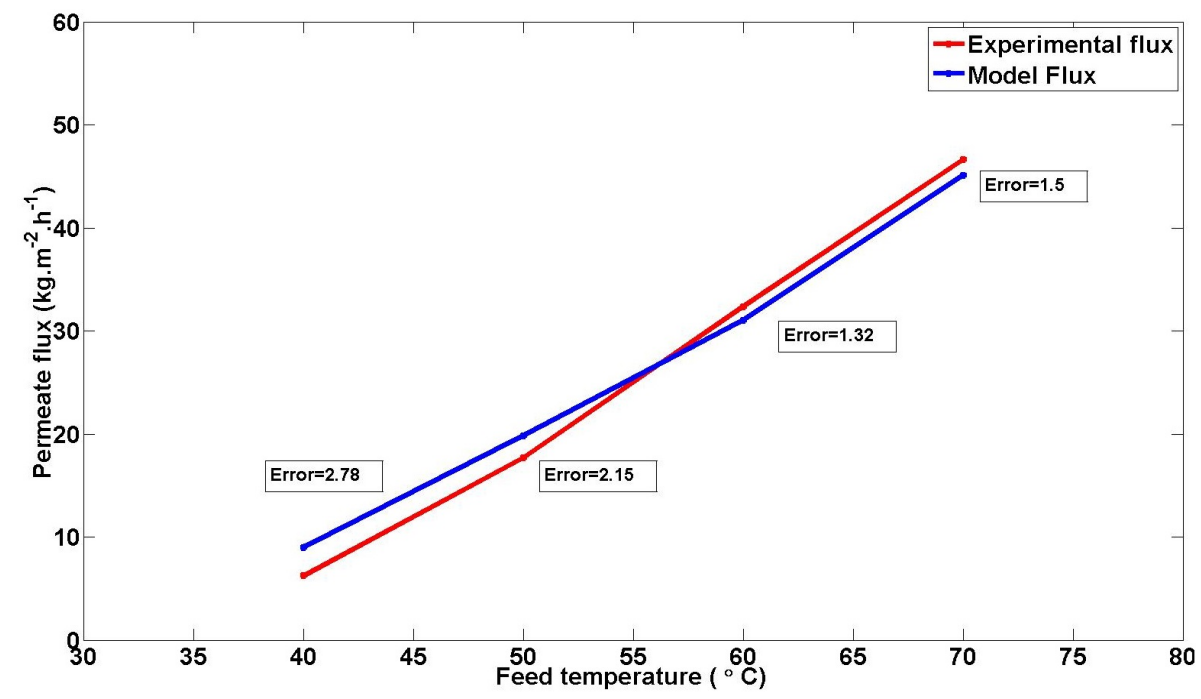

Figure 5: Validation of the dynamic model in the steady state phase, where feed inlet temperatures were set at $40^{\circ} \mathrm{C}, 50^{\circ} \mathrm{C}, 60^{\circ} \mathrm{C}, 70^{\circ} \mathrm{C}$. The maximum recorded absolute error is $2.78^{\circ} \mathrm{C}$.

The model acts best when compared to experiments at high feed inlet temperatures varying from $55^{\circ} \mathrm{C}$ to $70^{\circ} \mathrm{C}$, while the absolute error is a bit larger for lower temperatures below $40^{\circ} \mathrm{C}$. This is mainly due to the fact that, water vapor flux is higher at high feed temperatures, which reduces the errors in measurements compared to the very low flux obtained at low feed inlet temperatures. Additionally, the low sensitivity of the employed scale makes it difficult to record small production of permeate flux at low feed inlet temperatures.

\subsubsection{Time variation validation}

In the other set of experiments, the feed inlet temperature was increased in a ramp fashion from $30^{\circ} \mathrm{C}$ to $75^{\circ} \mathrm{C}$. The increment was $0.1^{\circ} \mathrm{C}$ every 2 min- 
utes, and the permeate flux as well as the outlet temperature were recorded. The comparison between the experiments and the model is shown in Fig.6.

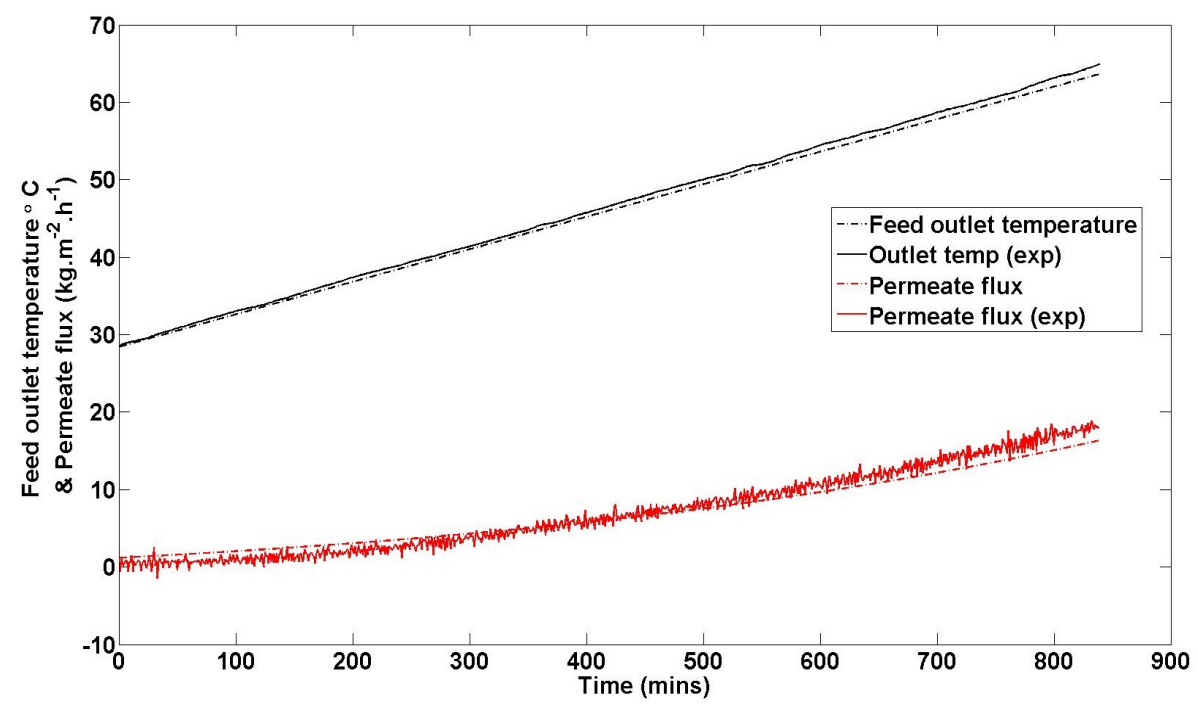

Figure 6: Validation of the dynamic model during time variation phase, where the feed inlet temperatures vary between $30^{\circ} \mathrm{C}$ and $75^{\circ} \mathrm{C}$ with $0.1^{\circ} \mathrm{C}$ increment every 2 minutes. Relative error doesn't exceed $\pm 5 \%$.

In this observation, the time variation response of the permeate flux and the outlet temperature show high consistency with the experimental data. This contributes towards the validity of the developed dynamic model to be extended to the time varying phase. Consequently, the validation in the steady state and the time variation phases emphasizes the direct proportional relationship between the permeate flux and the inlet temperatures, where it is known that higher feed inlet temperatures produce higher temperature on the boundary layers.

The MD process is highly sensitive to considerable variations in the membrane parameter values. For example, Fig. 7 shows the effect of the pore size variation on the membrane flux, where it is noticeable that the higher pore size leads to higher permeate flux. At the same time, the Liquid Entry Pressure $(L E P)$ should not be exceeded to ensure no pore wetting is occurring inside the membrane pores. The LEP is equal to 15 PSI for the used membrane, while the actual pressure difference at the liquid-vapor interface $\Delta P$ is lower or equal to 7.64-8.33 PSI for pore size equals to $0.5 \mu \mathrm{m}$ and along 
temperature variation between $\left(20^{\circ} \mathrm{C}-60^{\circ} \mathrm{C}\right)$, respectively. The variation of the membrane parameter values is very likely to happen when manufacturing the membranes, and so it leads introduce some divergence in some cases.

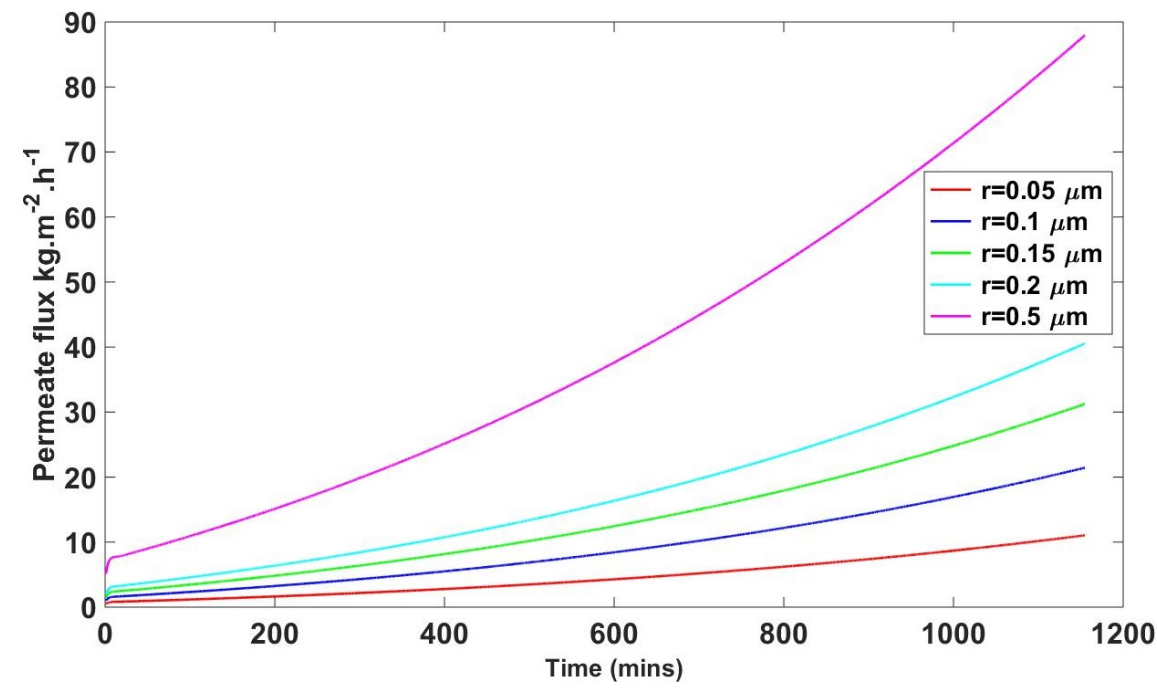

Figure 7: Effect of the variation of membrane pore size on permeate flux.

Experiment uncertainties can be reduced by using very sensitive temperature sensors and weighing scale, where small changes in feed temperature or permeate flux can be accurately detected. On the other hand, uncertainties in this study can come from the discretization scheme of the model, which can be reduced by refining the discretization grid.

\subsection{Model analysis}

The ADE model is applied inside the feed solution as well as at the permeate solution. For simulations and up-scaling purposes, software in MATLAB ${ }^{\circledR}$ environment is developed to solve the dynamic model and obtain behavior plots for analysis and improvement aspects.

Following simulations aim to highlight the capabilities of the dynamic model in both steady state and time varying phases. Therefore, real numerical values for the membrane parameters are used in the simulations as listed in Tables 1 and 3, besides feed and permeate inlet temperatures are set at $60{ }^{\circ} \mathrm{C}$ and $20^{\circ} \mathrm{C}$, respectively.

Fig. 8 shows the evolution of the feed and permeate solution temperatures after seconds of initiating the operation $(t=10 \mathrm{~s})$, where this phase repre- 
Table 3: Membrane distillation parameters.

\begin{tabular}{l|l|l|l}
\hline \hline Parameter & Value & Parameter & Value \\
\hline \hline Seawater thermal conductivity & $0.596 \mathrm{~W} / \mathrm{m} \cdot \mathrm{K}$ & Seawater density & $1035 \mathrm{~kg} / \mathrm{m}^{3}$ \\
Freshwater thermal conductivity & $0.607 \mathrm{~W} / \mathrm{m} \cdot \mathrm{K}$ & Freshwater density & $998.2 \mathrm{~kg} / \mathrm{m}^{3}$ \\
Flow rate of freshwater & $0.2 \mathrm{~m} / \mathrm{s}$ & Flow rate of seawater & $0.25 \mathrm{~m} / \mathrm{s}$ \\
Specific heat of freshwater & $3850 \mathrm{~J} / \mathrm{kg} \cdot{ }^{\circ} \mathrm{C}$ & Molecular weight of water & $18.01489 \mathrm{~g} / \mathrm{mol}$ \\
Specific heat of seawater & $4180 \mathrm{~J} / \mathrm{kg} \cdot{ }^{\circ} \mathrm{C}$ & $\begin{array}{l}\text { Average thermal conductivity } \\
\text { of membrane and vapor }\end{array}$ & $0.24 \mathrm{~W} / \mathrm{m} . \mathrm{K}$ \\
\hline \hline
\end{tabular}

sents the time varying phase. The figure shows the feed bulk temperature to be higher than the temperature at the membrane boundary layer from the feed side, and in a same manner the temperature of the membrane boundary layer from the permeate has higher temperature than permeate bulk. It also shows that the difference of temperature between the two sides of the membrane (driving force) is higher at the top side of the module (feed inlet side) and decreases gradually towards the bottom side (feed outlet side), which is in agreement with the heat transfer mechanisms. The corresponding permeate flux at that time instant is $29.15 \mathrm{~kg} / \mathrm{m}^{2}$.h. This phenomenon is further explained in [10].
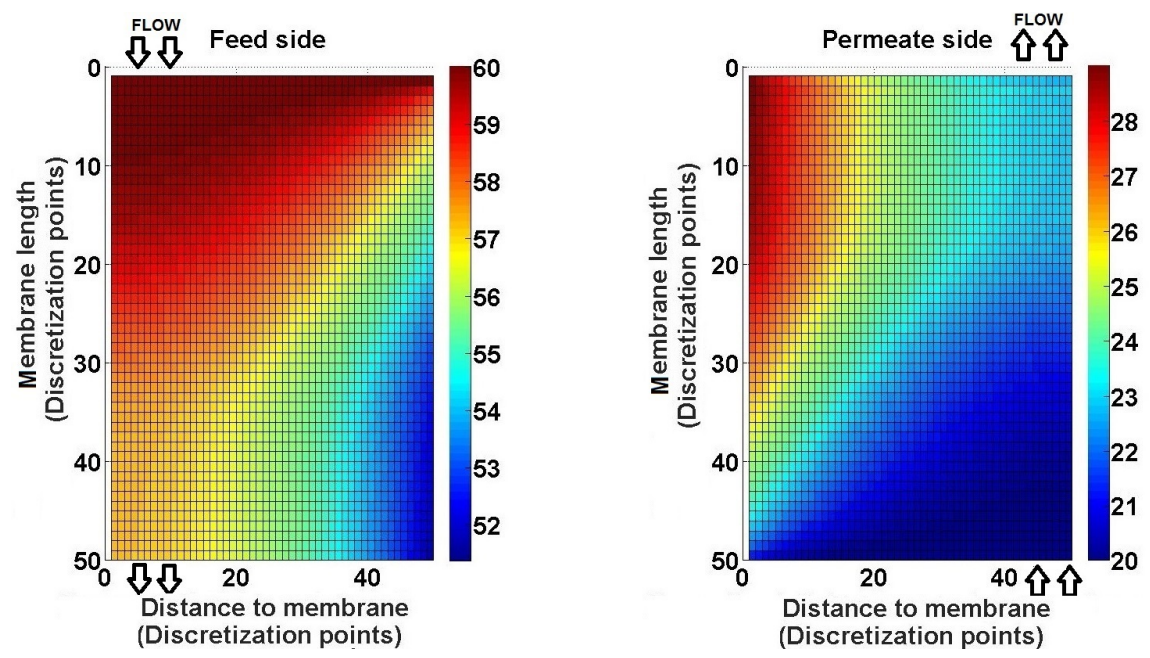

Figure 8: Evolution of feed and permeate solution temperatures after initiating the MD process at $t=10 \mathrm{~s}$ 
Fig.9 shows the temperature of the membrane boundary layers along the membrane normalized length at $t=10 \mathrm{~s}$. In the figure, the temperature at the top of the feed-membrane interface is close to $60^{\circ} \mathrm{C}$, and this is normal since the feed inlet is on the top, then the temperature on the feed-membrane interface decreases because of the heat transfer mechanisms. The same happens for the permeate-membrane interface. In the same context, the figure shows how close is the amount of temperature drop in the feed side and gained in the permeate side.

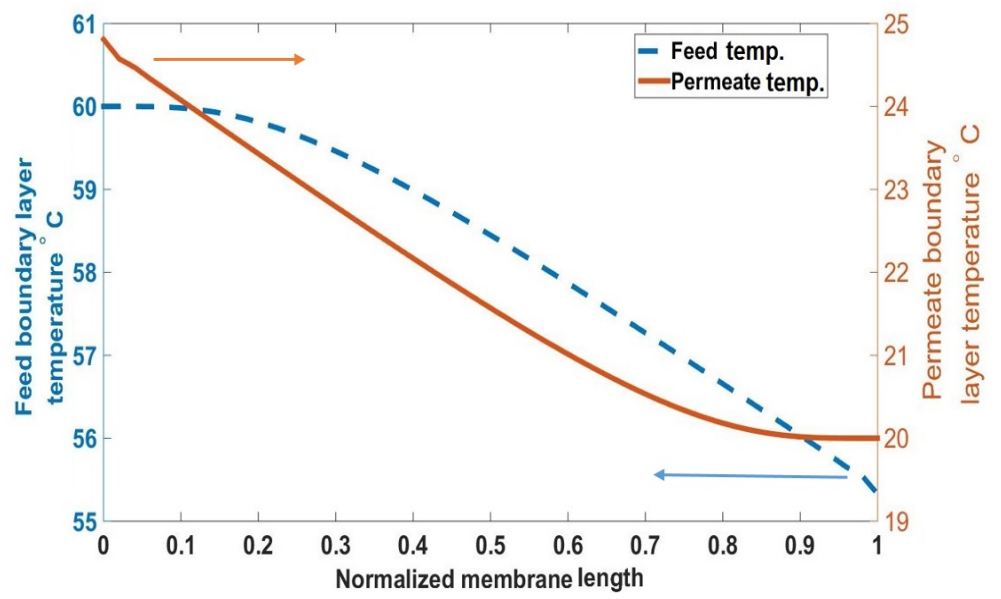

Figure 9: Evolution of temperature along the membrane length at $t=5$, where the feed interface (dashed curve) ranges between $55^{\circ} \mathrm{C}$ and $60^{\circ} \mathrm{C}$ and the permeate interface (solid curve) ranges between $20^{\circ} \mathrm{C}$ and $25^{\circ} \mathrm{C}$.

Similarly, the time evolution of the membrane boundary layers is shown in Fig.10. The figure shows the time evolution behavior of the boundary layers from the first seconds of running the operation until reaching the steady state phase. 

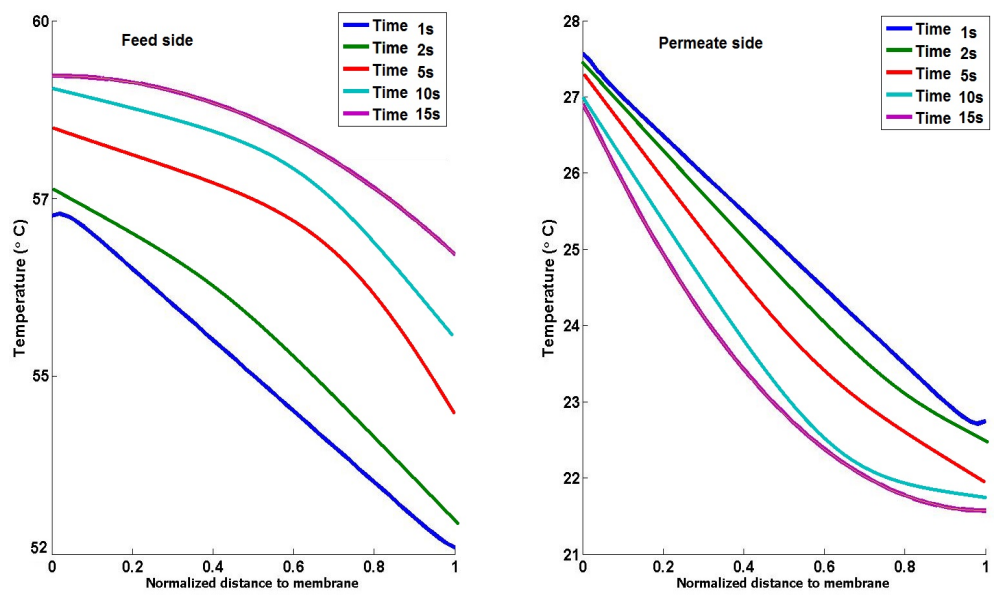

Figure 10: Time evolution of the membrane boundary layers at a middle point on the membrane module length.

The flow rate of the feed and the permeate streams have a significant role in managing the heat transfer inside the DCMD module. The flow rate controls the amount of the convective heat that transfers throughout the bulk solution, in which increasing its value makes the heat transfers via convection in the bulk more than the conduction through membrane pores. It is useful to have high convection effect, however continuous increasing in the flow rate is unprofitable because the heat flux would saturate eventually. A smart compromise should take place between convection and conduction mechanisms. The relation between the feed flow rate and the temperature for different flow rates in steady state phase is shown in Fig.11, where the simulation is captured on a point at the middle length of the membrane module and the operation lasted for 25 seconds to ensure reaching the steady state phase. Temperature of water molecules near the inlet increases rapidly while increasing the flow rate, and therefore, temperature polarization and residence time decrease, while the energy consumption increases. 

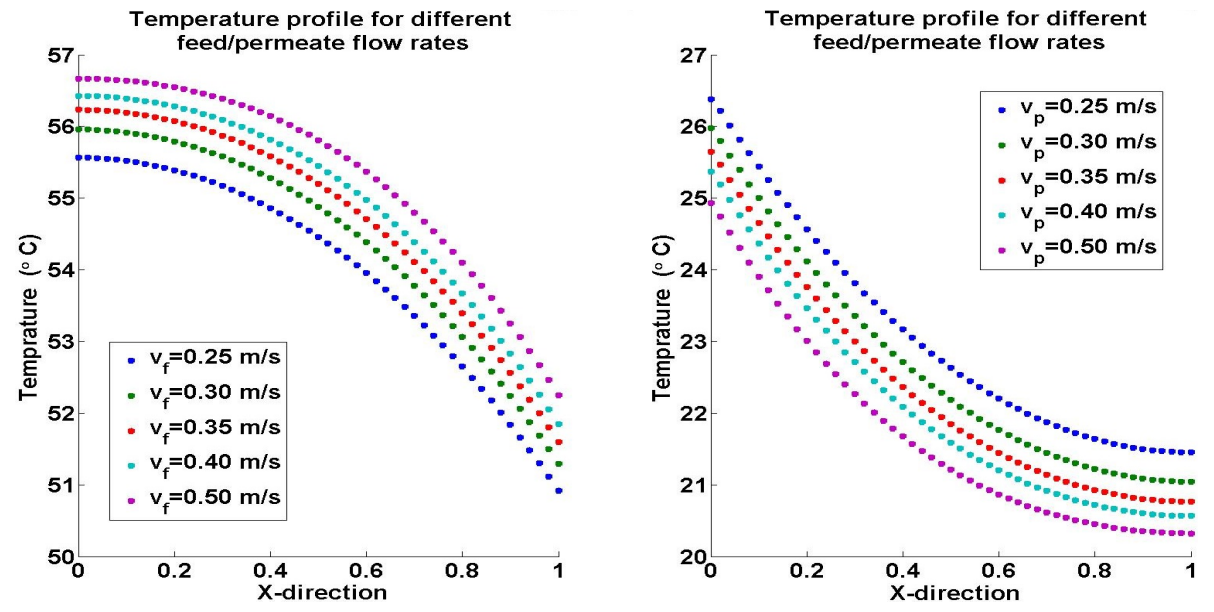

Figure 11: Effect of flow rate over temperature distribution.

The temperature polarization is simulated to check the time evolution of its coefficient (TPC). The formula of the TPC is shown in Eq.(26) $[3,46]$.

$$
T P C=\frac{T_{m f}-T_{m p}}{T_{b f}-T_{b p}},
$$

The value of TPC varies from zero for systems that suffer from large boundary layer resistances to unity for well designed systems. Fig.12 shows the evolution of TPC with time. During the transient phase of the process the amount of heat transfer through the membrane is low due to the starting of heat accumulation, and then it magnifies and increases after reaching steady state phase. 


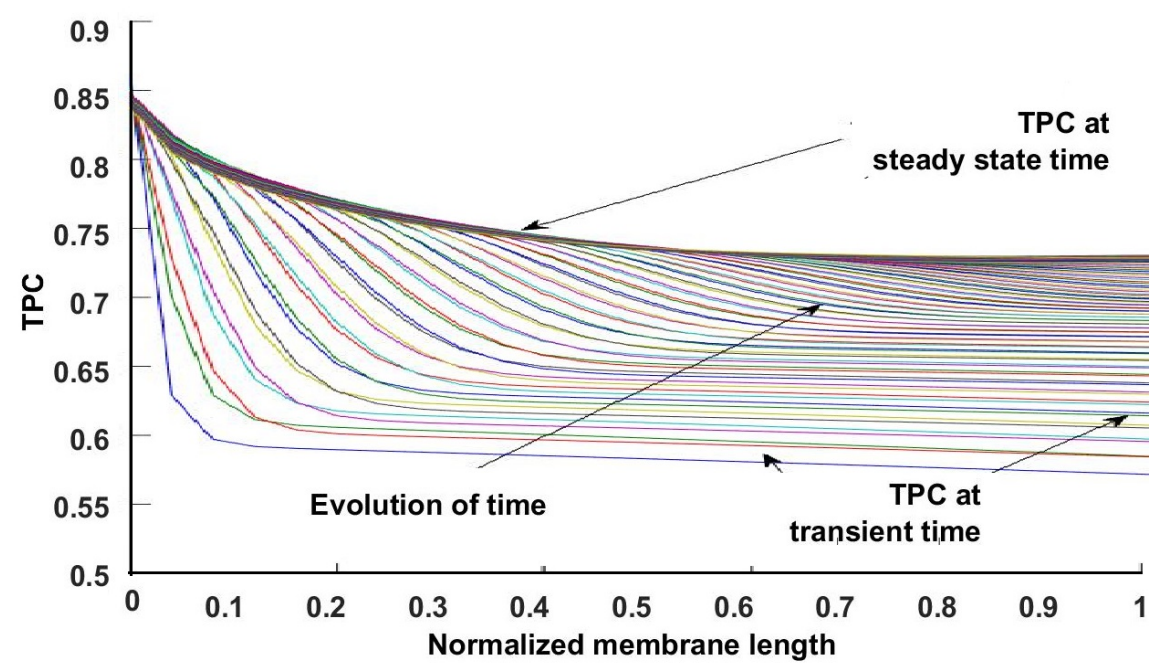

Figure 12: TPC evolution with time.

\section{Conclusions}

A mathematical dynamic model for DCMD process is reported in this manuscript. The mechanisms of heat and mass transfer that occur in the feed and permeate solutions are modeled with a 2D ADE model. The model is able to describe the behavior of the DCMD process in time variation phase as well as at steady state phase, and therefore paves for powering the DCMD process with intermittent energy supply, such as solar energy source efficiently. The model predictions are strongly correlated with the experiments in both steady state and time varying phases, with model predictions being within $\pm 5 \%$ of the experimentally obtained values. MATLAB ${ }^{\circledR}$ software is developed for simulating larger MD modules for scaling-up purposes.

\section{Acknowledgment}

The authors gratefully acknowledge that research reported in this publication was supported by the King Abdullah University of Science and Technology (KAUST).

\section{References}

[1] Y. D. Kim, K. Thu, N. Ghaffour, K. C. Ng, Performance investigation of a solar-assisted direct contact membrane distillation system, J. Membrane Sci. 427 (0) (2013) 345 - 364. 
[2] A. Alkhudhiri, N. Darwish, N. Hilal, Membrane distillation: A comprehensive review, Desalination 287 (0) (2012) 2 - 18, special Issue in honour of Professor Takeshi Matsuura on his 75th Birthday.

[3] M. Khayet, Membranes and theoretical modeling of membrane distillation: A review, Advances in Colloid and Interface Science 164 (12) (2011) 56 - 88, membrane Separation and Colloid Science.

[4] M. Khayet, Advanced Membrane Technology and Applications, John Wiley \& Sons, Inc., 2008.

[5] A. S. Alsaadi, L. Francis, G. L. Amy, N. Ghaffour, Experimental and theoretical analyses of temperature polarization effect in vacuum membrane distillation, J. Membrane Sci. 471 (0) (2014) 138 - 148.

[6] K. W. Lawson, D. R. Lloyd, Membrane distillation, J. Membrane Sci. 124 (1) (1997) $1-25$.

[7] K. Charfi, M. Khayet, M. Safi, Numerical simulation and experimental studies on heat and mass transfer using sweeping gas membrane distillation, Desalination 259 (13) (2010) $84-96$.

[8] T. M. Missimer, Y.-D. Kim, R. Rachman, K. C. Ng, Sustainable renewable energy seawater desalination using combined-cycle solar and geothermal heat sources, Desalination and Water Treatment 51 (4-6) (2013) 1161-1170.

[9] N. Ghaffour, S. Lattemann, T. Missimer, K. C. Ng, S. Sinha, G. Amy, Renewable energy-driven innovative energy-efficient desalination technologies, Applied Energy 136 (0) (2014) 1155 - 1165.

[10] L. Francis, N. Ghaffour, A. Alsaadi, S. Nunes, G. Amy, Performance evaluation of the DCMD desalination process under bench scale and large scale module operating conditions, J. Membrane Sci. 455 (2014) $103-112$.

[11] M. Gryta, Water Desalination by Membrane Distillation, Desalination, Trends and Technologies, Michael Schorr (Ed.), InTech.

[12] M. Khayet, T. Matsuura, Chapter 10 - Direct Contact Membrane Distillation, Elsevier, Amsterdam, 2011. 
[13] L. Francis, N. Ghaffour, A. A. Alsaadi, G. L. Amy, Material gap membrane distillation: A new design for water vapor flux enhancement, J. Membrane Sci. 448 (0) (2013) 240 - 247.

[14] M. Ali, H. Fath, P. Armstrong, A comprehensive techno-economical review of indirect solar desalination, Renewable and Sustainable Energy Reviews 15 (8) (2011) 4187 - 4199.

[15] M. Gryta, M. Tomaszewska, Heat transport in the membrane distillation process, J. Membrane Sci. 144 (12) (1998) 211 - 222.

[16] L. Martnez-Dez, M. Vzquez-Gonzlez, Temperature and concentration polarization in membrane distillation of aqueous salt solutions, J. Membrane Sci. 156 (2) (1999) 265 - 273.

[17] F. Eleiwi, T. M. Laleg, Dynamic modeling and optimization in membrane distillation system, The 19th World Congress of the International Federation of Automatic Control (2014) 3327-3332.

[18] F. Eleiwi, T. M. Laleg-Kirati, Membrane distillation process modeling: Dynamical approach, International Journal of Chemical, Nuclear, Metallurgical and Materials Engineering 8 (6) (2014) 479 - 484.

[19] D. Jähnig, R. Hausner, W. Wagner, C. Isaksson, Thermo-chemical storage for solar space heating in single-family house, New Jersey, 2006.

[20] A. Sharma, V. Tyagi, C. Chen, D. Buddhi, Review on thermal energy storage with phase change materials and applications, Renewable and Sustainable Energy Reviews 13 (2) (2009) 318 - 345.

[21] B. Mette, H. Kerskes, H. Drck, Concepts of long-term thermochemical energy storage for solar thermal applications selected examples, Energy Procedia 30 (0) (2012) 321 - 330, 1st International Conference on Solar Heating and Coolingfor Buildings and Industry (SHC 2012).

[22] F. Eleiwi, T. Laleg-Kirati, Nonlinear Lyapunov-based boundary control of distributed heat transfer mechanisms in membrane distillation plant, American Control Conference (ACC) (2015) 4964-4969. 
[23] F. Eleiwi, I. N'Doye, T.-M. Laleg-Kirati, Feedback control for distributed heat transfer mechanisms in direct-contact membrane distillation system, Control Applications (CCA), 2015 IEEE Conference on (2015) 1624-1629.

[24] J. Zhang, Theoretical and experimental investigation of membrane distillation, Ph.D. thesis, Victoria University (2011).

[25] R. Schofield, A. Fane, C. Fell, Gas and vapour transport through microporous membranes. ii. membrane distillation, J. Membrane Sci. 53 (12) (1990) $173-185$.

[26] R. Schofield, Heat and mass transfer in membrane distillation, J. Membrane Sci. 33 (3) (1987) 299-313.

[27] N. Li, A. Fane, W. Ho, T. Matsuura, Advanced Membrane Technology and Applications, Wiley, 2011.

[28] P. Termpiyakul, R. Jiraratananon, S. Srisurichan, Heat and mass transfer characteristics of a direct contact membrane distillation process for desalination, Desalination 177 (1-3) (2005) 133-141.

[29] M. Khayet, Study of Asymmetric Polarization in Direct Contact Membrane Distillation, Separation Science and Technology 39 (1) (2005) 125147.

[30] H. Yu, X. Yang, R. Wang, A. G. Fane, Analysis of heat and mass transfer by CFD for performance enhancement in direct contact membrane distillation, J. Membrane Sci. 405 - 406 (0) (2012) 38 - 47.

[31] L.-Z. Zhang, C.-H. Liang, L.-X. Pei, Conjugate heat and mass transfer in membrane-formed channels in all entry regions, International Journal of Heat and Mass Transfer 53 (5-6) (2010) 815-824.

[32] N. Tang, H. Zhang, W. Wang, Computational fluid dynamics numerical simulation of vacuum membrane distillation for aqueous nacl solution, Desalination 274 (13) (2011) 120 - 129.

[33] A. Cipollina, G. Micale, L. Rizzuti, Membrane distillation heat transfer enhancement by cfd analysis of internal module geometry, Desalination and Water Treatment 25 (1-3) (2011) 195-209. 
[34] M. Ghadiri, S. Fakhri, S. Shirazian, Modeling and cfd simulation of water desalination using nanoporous membrane contactors, Industrial \& Engineering Chemistry Research 52 (9) (2013) 3490-3498.

[35] A. Alklaibi, N. Lior, Transport analysis of air-gap membrane distillation, J. Membrane Sci. 255 (12) (2005) 239 - 253.

[36] S. Bouguecha, R. Chouikh, M. Dhahbi, Numerical study of the coupled heat and mass transfer in membrane distillation, Desalination 152 (13) (2003) $245-252$.

[37] S. Al-Sharif, M. Albeirutty, A. Cipollina, G. Micale, Modelling flow and heat transfer in spacer-filled membrane distillation channels using open source CFD code, Desalination 311 (2013) 103 - 112.

[38] M. Shakaib, S. Hasani, I. Ahmed, R. M. Yunus, A CFD study on the effect of spacer orientation on temperature polarization in membrane distillation modules, Desalination 284 (2012) 332 - 340.

[39] M. Shakaib, S. Hasani, M. E. ul Haque, I. Ahmed, R. Yunus, A cfd study of heat transfer through spacer channels of membrane distillation modules, Desalination and Water Treatment 51 (16-18) (2013) 36623674 .

[40] J. Santos, V. Geraldes, S. Velizarov, J. Crespo, Investigation of flow patterns and mass transfer in membrane module channels filled with flow-aligned spacers using computational fluid dynamics CFD, J. Membrane Sci. 305 (12) (2007) 103 - 117.

[41] R. Hetnarski, M. Eslami, Thermal Stresses - Advanced Theory and Applications, Solid Mechanics and Its Applications, Springer Netherlands, 2008 .

[42] J. Unsworth, F. J. Duarte, Heat diffusion in a solid sphere and fourier theory: An elementary practical example, American Journal of Physics 47 (11).

[43] Y. Jeon, Hybridized SUPG and upwind numerical schemes for convection dominated diffusion problems, Journal of Computational and Applied Mathematics 275 (0) (2015) 91 - 99. 
[44] M. Valipour, A. A. Montazar, Sensitive analysis of optimized infiltration parameters in swdc model, Advances in Environmental Biology 6 (9) (2012) 2574-2581.

[45] M. Valipour, A. A. Montazar, An evaluation of swdc and winsrfr models to optimize of infiltration parameters in furrow irrigation, American Journal of Scientific Research 69 (2012) 128-142.

[46] L. Martinez-Diez, Study of evaporation efficiency in membrane distillation, Desalination 126 (1999) 193-198. 